\title{
Supplementary Materials: Strain depth profiles in thin films extracted from in-plane X-ray diffraction
}

\author{
C. Cancellieri, ${ }^{1, *}$ D. Ariosa ${ }^{2}$ A. V. Druzhinin, ${ }^{1,3}$ Y. \\ Unutulmazsoy, ${ }^{1}$ A. Neels, ${ }^{4}$ and L. P.H. Jeurgens ${ }^{1}$ \\ ${ }^{1}$ Empa, Swiss Federal Laboratories for Materials Science and Technology, \\ Laboratories for Joining Technologies and Corrosion, \\ Überlandstrasse 129, 8600 Dübendorf, Switzerland \\ ${ }^{2}$ Instituto de Física, Facultad de Ingeniería, \\ Universidad de la República, Herrera y Reissig 565, \\ C.C. 30, 11000 Montevideo, Uruguay \\ ${ }^{3}$ National University of Science and Technology MISIS, \\ Leninsky prospect 4, Moscow 119049, Russian Federation \\ ${ }^{4}$ Empa, Swiss Federal Laboratories for Materials Science and Technology, \\ Center for X-ray Analytics, Department Materials Meet Life, \\ Überlandstrasse 129, 8600 Dübendorf, Switzerland
}

(Dated: November 2, 2020)

*Electronic address: claudia.cancellieri@empa.ch 


\section{A. Peak fit}

In this section an example of peak fitting is shown. The fit used to derive the peak position in the analysis was a Gaussian function. The example in Fig. S1 displays the fit on the $\mathrm{Cu}(220)$ peak of a $300 \mathrm{~nm}$ film measured in the IP-GID geometry at $\alpha=0.2^{\circ}$.

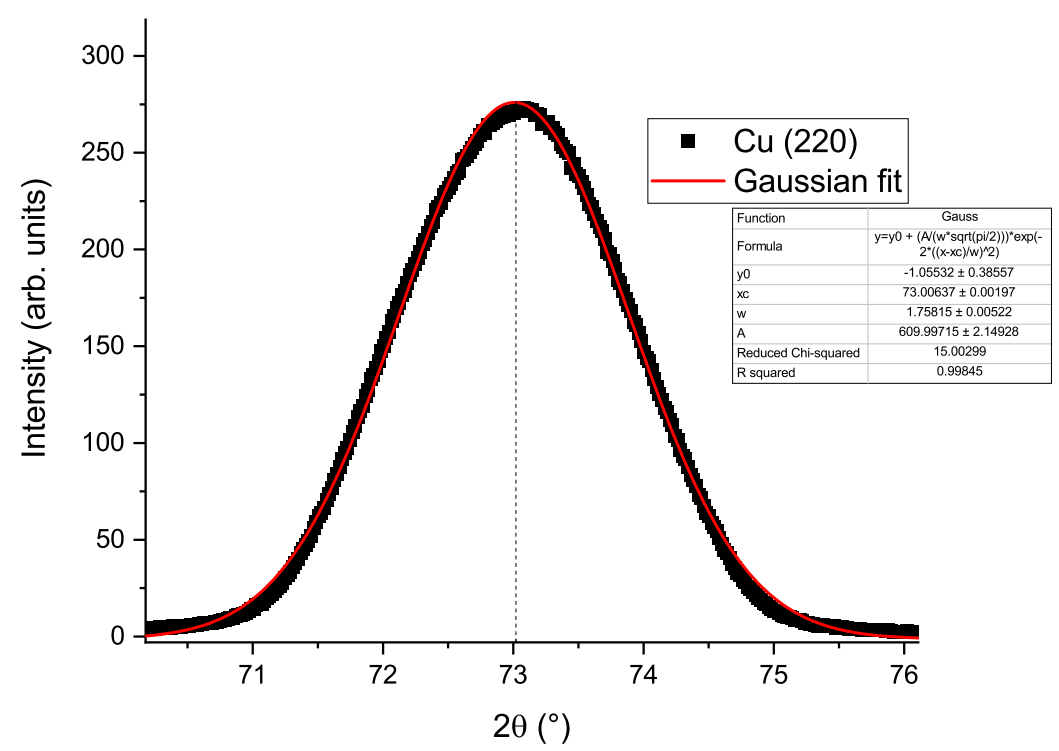

FIG. S1: (Color online) Gaussian fit on the $\mathrm{Cu}(220)$ reflection measured on the $300 \mathrm{~nm}$ thick film.

\section{B. Fit results for 20,150 and $300 \mathrm{~nm} \mathrm{Cu}$}

In this section we present the fit results using the 4 different methods described in the main text applied to 20 (Fig. S2), 150 (Fig. S3) and 300 (Fig. S4) nm Cu thick films. 
a)

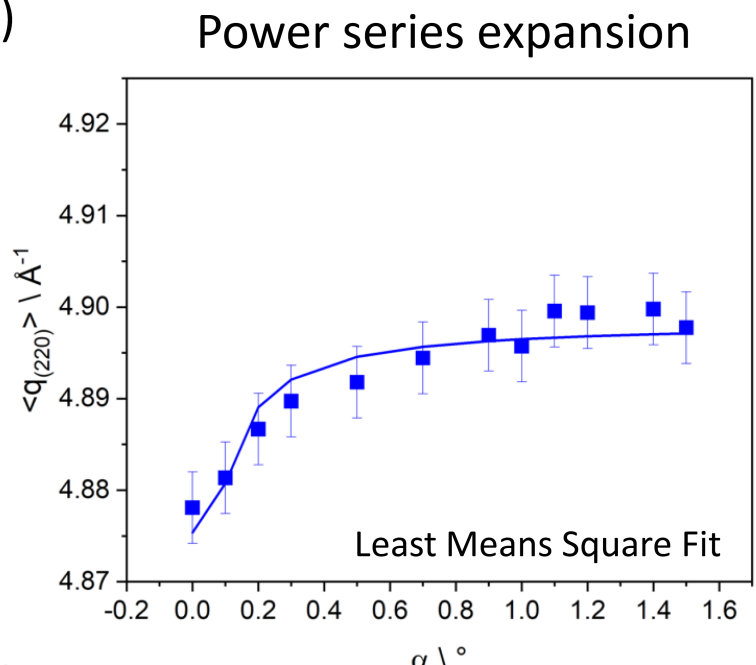

b)

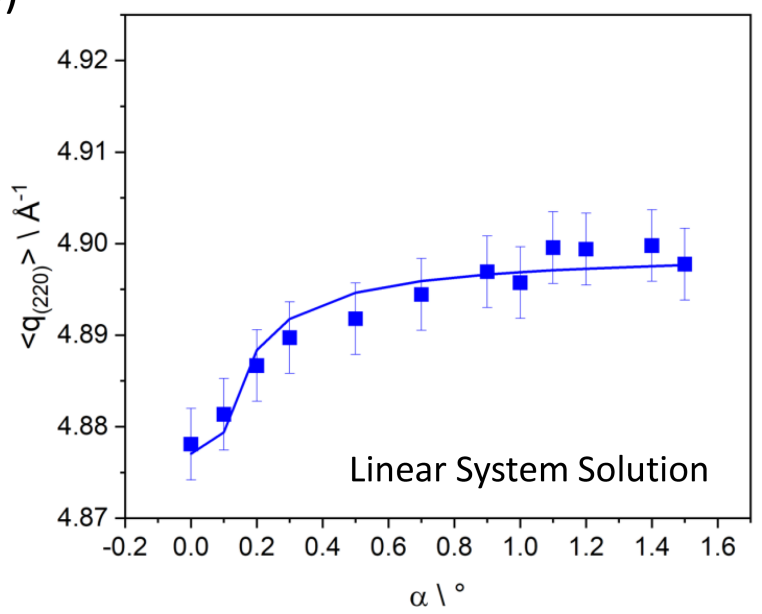

$20 \mathrm{~nm}$

c) Fourier series expansion

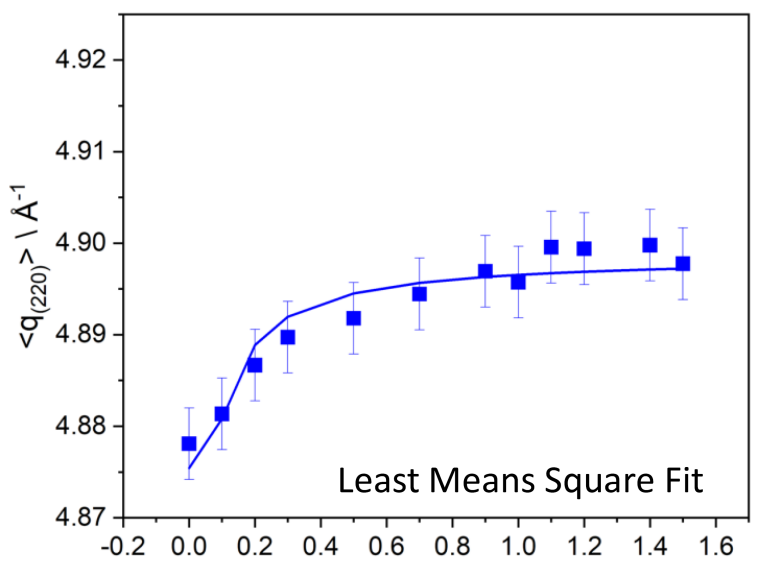

d) $\alpha \backslash^{\circ}$

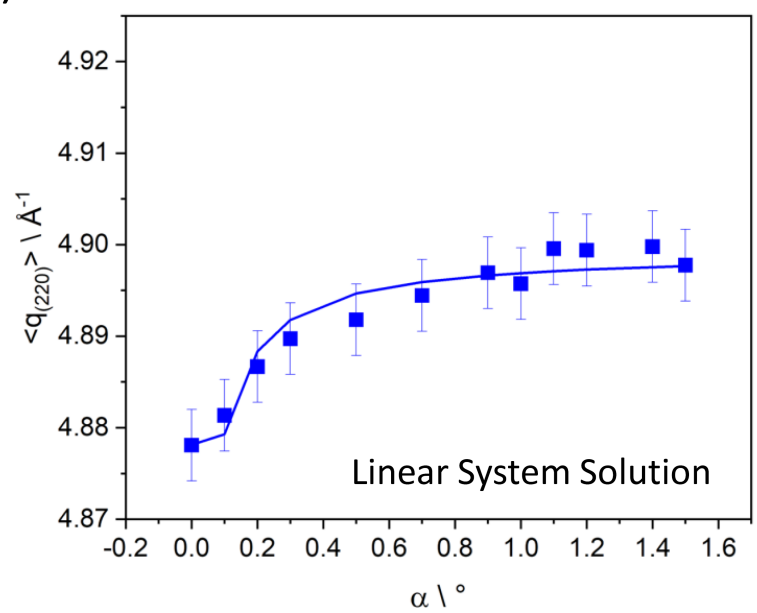

FIG. S2: (Color online) Cu $20 \mathrm{~nm}$ sample. Fit of the average peak position for the (220) Bragg reflexion as a function of the incident angle. Montecarlo least squares fit a) for power series expansion and c) for Fourier series expansion. Linear system solution b) for power series expansion and d) for Fourier series expansion.

All the obtained fits independently of the method, produced a similar strain profile for each thickness, reported in the main text. 


\section{$150 \mathrm{~nm}$}

a)

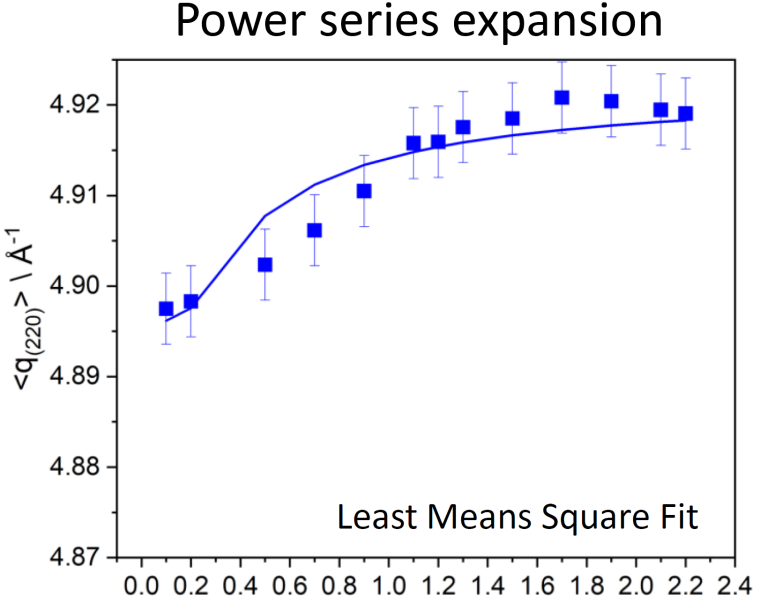

b)

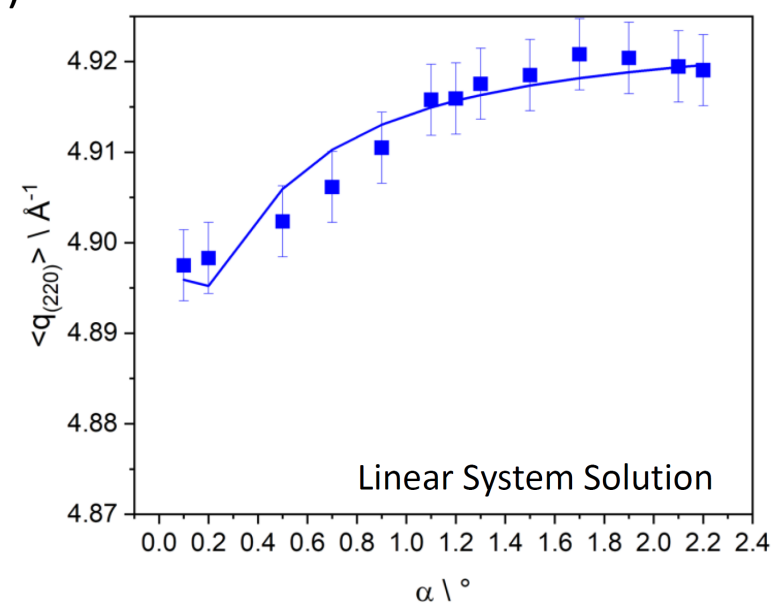

c) Fourier series expansion

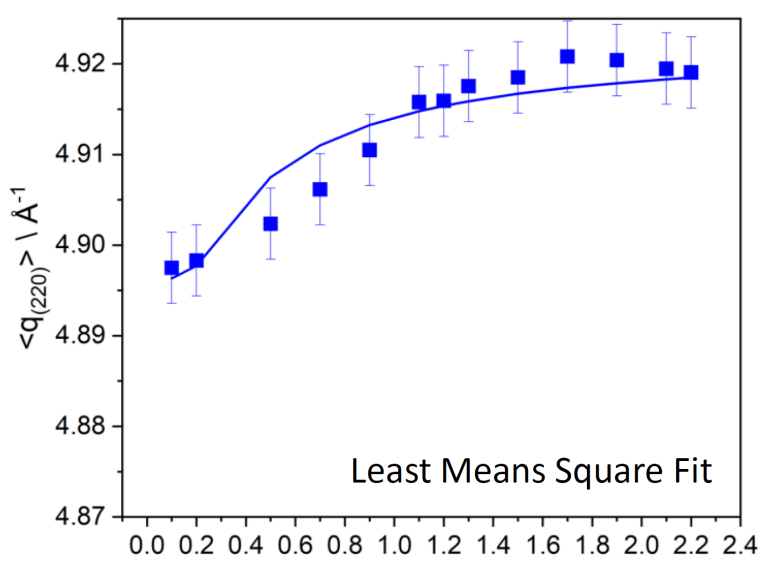

d)

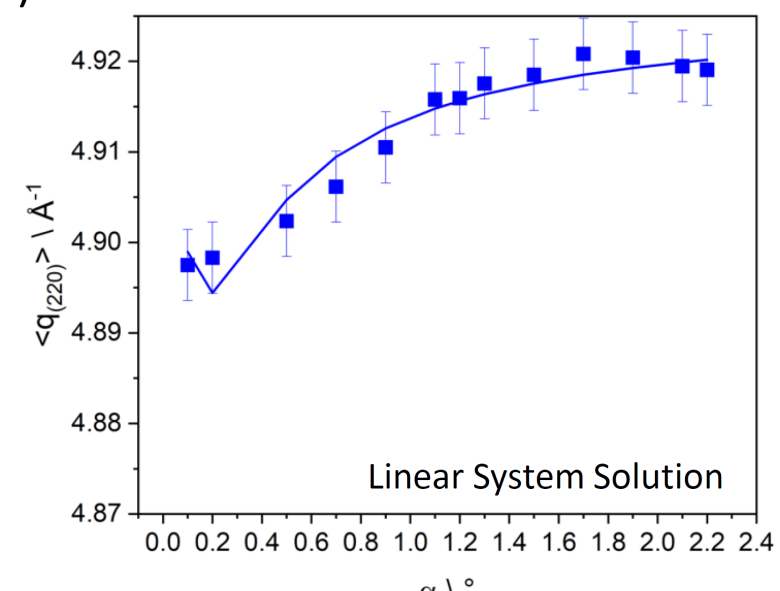

FIG. S3: (Color online) $\mathrm{Cu} 150 \mathrm{~nm}$ sample. Fit of the average peak position for the (220) Bragg reflexion as a function of the incident angle. Montecarlo least squares fit a) for power series expansion and c) for Fourier series expansion. Linear system solution b) for power series expansion and d) for Fourier series expansion.

\section{Cu morphology}

The surface morphology of metal and reduced $\mathrm{Cu}$ films was characterized by a high resolution Scanning electron microscope (SEM, Hitachi S-4800). The clear difference in morphology between the compact as deposited $\mathrm{Cu}$ thin film and the porous $\mathrm{Cu}$ reduced film is depicted in Fig.S5. 


\section{$300 \mathrm{~nm}$}

a)

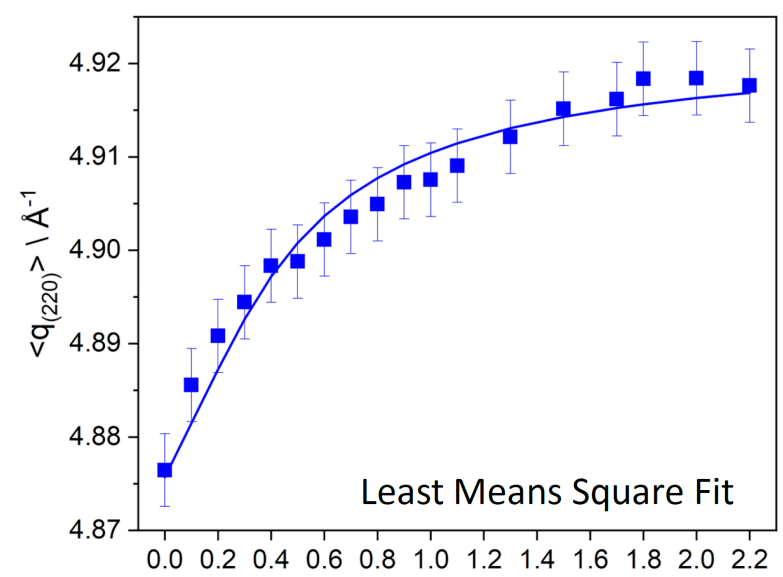

b)

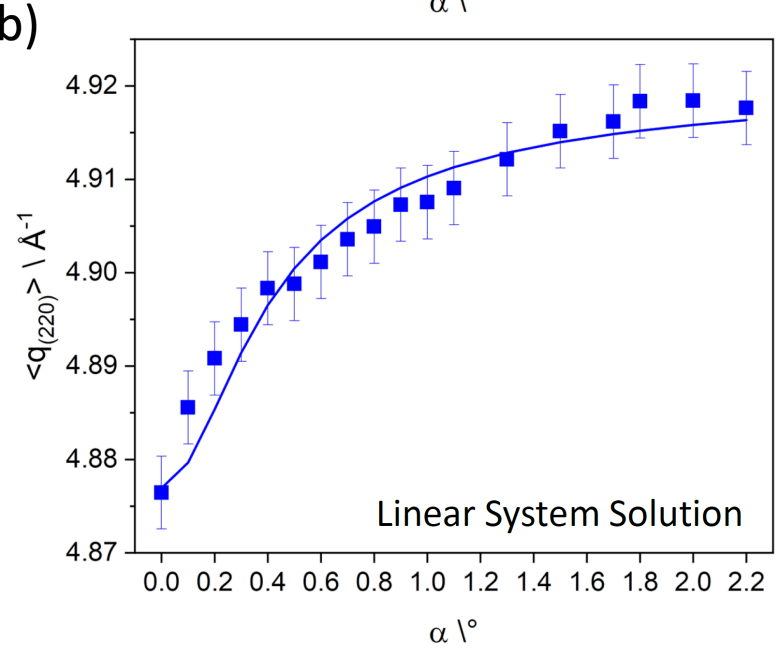

c) Fourier series expansion

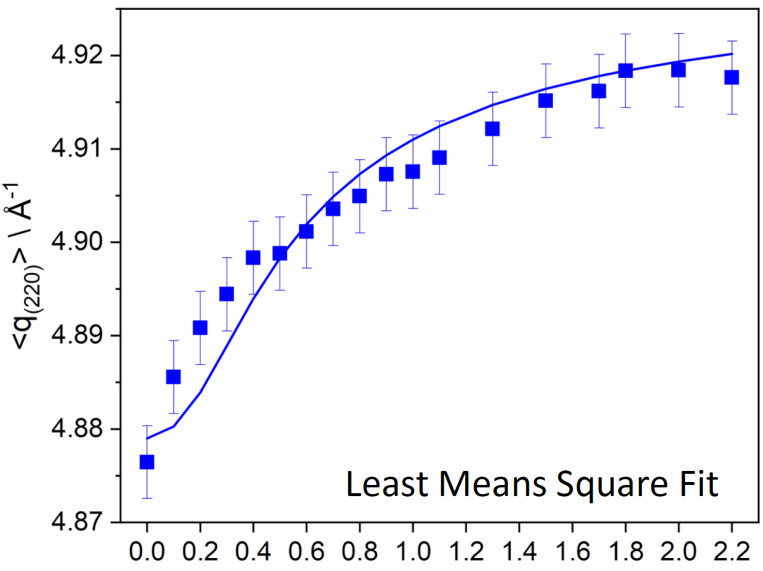

d)

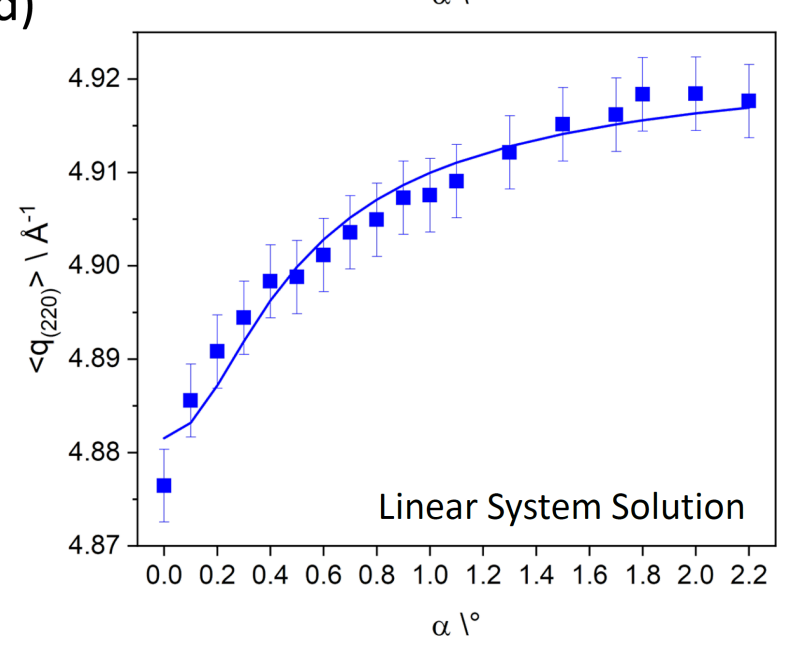

FIG. S4: (Color online) $\mathrm{Cu} 300 \mathrm{~nm}$ sample. Fit of the average peak position for the (220)

Bragg reflexion as a function of the incident angle. Montecarlo least squares fit a) for power series expansion and c) for Fourier series expansion. Linear system solution b) for power series expansion and d) for Fourier series expansion. 
a)

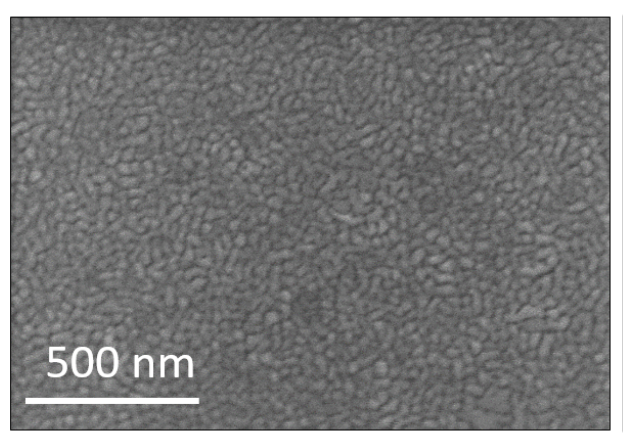

b) reduced $\mathrm{Cu}$

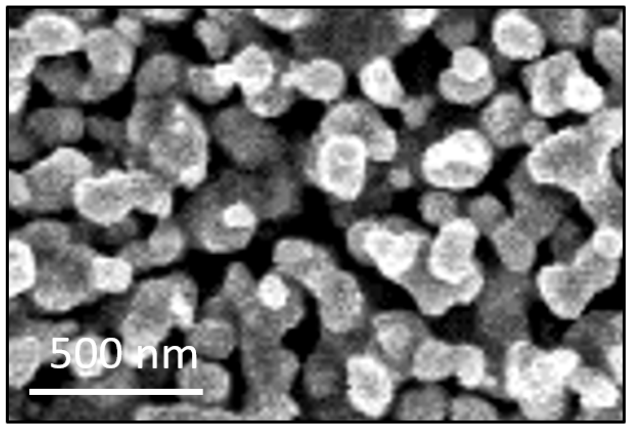

FIG. S5: (Color online) a) Typical Cu metallic thin film morphology and b) porous structure after oxidation and reduction process measured for a $300 \mathrm{~nm} \mathrm{Cu}$ film. 